\title{
Spontaneous bacterial peritonitis and pneumonia caused by Bordetella bronchiseptica
}

\author{
Nomonde R Dlamini', Ahmed Bhamjee ${ }^{1}$, Penelope Levick ${ }^{1}$, Evelyn Uniacke ${ }^{1}$, Husna Ismail ${ }^{2}$, \\ Anthony M Smith ${ }^{2}$ \\ ${ }^{1}$ Department of Clinical Microbiology, National Health Laboratory Service, Addington Hospital, Durban, South Africa \\ ${ }^{2}$ Enteric Diseases Reference Unit, National Institute for Communicable Diseases a division of the National Health \\ Laboratory Service, Johannesburg, South Africa
}

\begin{abstract}
Bordetella bronchiseptica is a rare cause of invasive human infection. The most common infection in humans is the respiratory tract infection and it is usually associated with immunosuppression, particularly acquired immunodeficiency syndrome (AIDS). We report a case of a pneumonia and peritonitis in a 42-year-old female with alcoholic liver disease. The patient died despite treatment with antibiotics. This case illustrates the potential virulence of $B$. bronchiseptica in susceptible patients and to our knowledge it is the first case of primary peritonitis due to this organism.
\end{abstract}

Key words: Bordetella bronchiseptica; peritonitis; pneumonia

J Infect Dev Ctries 2012; 6(7):588-591.

(Received 09 May 2011 - Accepted 14 September 2011)

Copyright $(0) 2012$ Dlamini et al. This is an open-access article distributed under the Creative Commons Attribution License, which permits unrestricted use, distribution, and reproduction in any medium, provided the original work is properly cited.

\section{Introduction}

Bordetella bronchiseptica is a pleomorphic Gram-negative coccobacillus that is found as a commensal in the upper respiratory tracts of wild and domestic animals where it may cause infection [1]. The well-described animal infections are the infectious tracheobronchitis in dogs and atrophic rhinitis in pigs [1]. Humans can become colonized in the respiratory tract, which makes interpretation of results difficult if the organism is isolated from the respiratory tract secretions. Human infections rarely occur, the most common being respiratory tract infections [1]. We present a case of severe pneumonia with peritonitis in a patient with alcoholic liver disease.

\section{Case report}

A 42-year-old female presented with a threeweek history of abdominal distention and vomiting. There was no history of fever. Other complaints were loss of weight, loss of appetite and painful feet. The patient had a strong history of alcohol abuse and smoking. She drank about two liters of wine daily for more than fifteen years and she had a twenty- pack/year history of smoking. The patient stayed with a partner and she was unemployed. There was no history of travel. On further inquiry there was no history of exposure to animals. The patient had been feeling unwell for four months but had never presented to hospital prior to this. No significant past medical history was elicited except that she was allergic to penicillin.

On examination the patient appeared drowsy and lethargic. Her temperature was $36.5^{\circ} \mathrm{C}$; Blood pressure was $110 / 80 \mathrm{mmHg}$; and pulse rate was 110 per minute and regular. There was bipedal pitting oedema up to the knee. The patient was jaundiced. There were cutaneous signs of chronic liver disease which included spider naevi, palmar erythema and axillary alopecia. The abdominal examination revealed distended abdomen with visible veins. The abdomen was diffusely tender. A fluid thrill was elicited suggestive of massive ascites. The examination for organomegaly was unsuccessful because the abdomen was very tense due to ascites. The ultrasound of the abdomen showed massive ascites and an $18 \mathrm{~cm}$ hepatomegaly. Examination of the respiratory system revealed mild tachypnoea with 
good bilateral air entry. There were no crepitations or any other abnormal sounds. The cardiovascular examination showed no abnormality. The initial diagnosis was that of chronic liver disease secondary to alcohol abuse with grade 1 hepatic encephalopathy.

Laboratory investigations showed haemoglobin of $10.8 \mathrm{~g} / \mathrm{dl}$; leucocytes of $13.5 \times 10^{9} / \mathrm{L}$; platelets $224 \times 10^{9} / \mathrm{L}$; International normalized ratio 1.42 ; urea nitrogen $4.8 \mu \mathrm{mol} / \mathrm{L}$; creatinine $121 \mu \mathrm{mol} / \mathrm{L}$; alkaline phosphatase $311 \mu \mathrm{mol} / \mathrm{L}$; total bilirubin $93 \mu \mathrm{mol} / \mathrm{L}$; conjugated bilirubin $46 \mu \mathrm{mol} / \mathrm{L}$; albumin $11 \mathrm{~g} / \mathrm{L}$; total protein $58 \mathrm{~g} / \mathrm{L} ;$ gamma-glutamyl transpeptidase $182 \mathrm{u} / \mathrm{L}$ and alanine transaminase $71 \mathrm{u} / \mathrm{L}$. The aspartate transaminase test was not done. The hepatitis B surface antigen was negative. The tumor marker alpha fetoprotein and carcinoembryonic antigen were normal. The human immunodeficiency virus (HIV) rapid antibody test was negative.

An ascitic tap was also done and it showed glucose of $5.7 \mathrm{mmol} / \mathrm{L}$ and a transudate fluid as evidenced by very low LDH of $81 \mathrm{U} / \mathrm{L}$ and protein of $3 \mathrm{~g} / \mathrm{L}$. The blood glucose was $6.1 \mathrm{mmol} / \mathrm{L}$. The cell count was not done but the Gram stain showed few pus cells and no organisms. The culture of the peritoneal fluid grew Gram-negative coccobacilli on chocolate, blood and MacConkey agars incubated at $37^{\circ} \mathrm{C}$ aerobically. The organism was positive for oxidase and catalase and was identified as $B$. bronchiseptica by the Vitek II compact system (bioMeriux, Marcy-I'Etoile, France). The results of the susceptibility testing with an AST-N133 kit, from the Vitek II compact system (bioMeriux, MarcyI'Etoile, France) showed resistance to trimethoprim/sulfamethoxazole, ampicillin, cefuroxime, cefotaxime and cefoxitin; intermediate resistance to ceftazidime and cefepime; and sensitivity to meropenem, piperacillin/tazobactam, amoxicillin/clavulanic acid, amikacin, gentamicin, ciprofloxacin, tigecycline and colistin. The identity of the organism was confirmed using 16SrRNA gene sequencing technology. The nucleotide sequence of the 16SrRNA gene was examined against the DNA database of the National Center for Biotechnology Information using the BLAST algorithm [2] and the best alignments were obtained against $B$. bronchiseptica sequences.

The initial chest $X$ ray performed on admission showed opacification on the right upper lobe; the subsequent chest $\mathrm{x}$-ray taken three days later showed worsening with involvement of almost the entire right lung (Figure 1). Throughout the hospital stay, the patient remained apyrexial with no change in the clinical condition. A sputum sample taken a day after the second chest $\mathrm{X}$ ray grew $B$. bronchiseptica with the same susceptibility as the one isolated from the ascitic fluid. No blood cultures were taken.

The patient was started on ciprofloxacin $400 \mathrm{mg} / 12 \mathrm{hr}$ intravenously, and the ascitic fluid was drained. Despite treatment, her condition did not improve. On the third day after commencement of antibiotic therapy the patient suddenly developed severe respiratory distress while sitting on a chair. Attempts at resuscitation in the ward were unsuccessful and the patient died.

\section{Discussion}

Bordetella bronchiseptica is a rare cause of invasive human infection. Human infections are usually associated with immunosuppression, particularly acquired immunodeficiency syndrome (AIDS) [3]. Immunosuppression due to alcoholic liver disease was the most likely predisposing factor for the infection in this patient. A history of contact with animals is helpful, but if negative it does not exclude $B$. bronchiseptica infection [4].

The pneumonia was an incidental finding diagnosed on routine chest $\mathrm{X}$ ray as the patient did not present with any respiratory symptoms. In 1979, H. K. Ghosh and J. Tranter [5] reported a 73-year-old man with alcoholic malnutrition who presented with severe respiratory infection and septicaemia caused by $B$. Bronchiseptica. The $\mathrm{X}$ ray changes showed sudden deterioration and the patient died three days later. These findings highlight the potentially aggressive nature of this organism in susceptible patients. In our patient, isolates from the sputum and from the ascitic fluid were identical. We therefore speculate that the infection probably started in the respiratory tract and spread to the peritoneum through the bloodstream, even though the blood cultures were never taken. The liver is a major draining site of blood from the systemic circulation. Bacteria present in the blood can then contaminate the hepatic lymph and pass through the lymphatic walls into the ascitic fluid causing infection [6]. The impairment of the hepatic reticuloendothelial system in alcoholic liver disease would favour the establishment of this infection [7].

Although peritonitis in patients on continuous ambulatory peritoneal dialysis has been previously reported in the literature [4,8-9], to our knowledge, this is the first case of spontaneous bacterial peritonitis caused by $B$. bronchiseptica. This 
Figure 1. Chest $\mathrm{X}$ ray showing opacification of the right lung

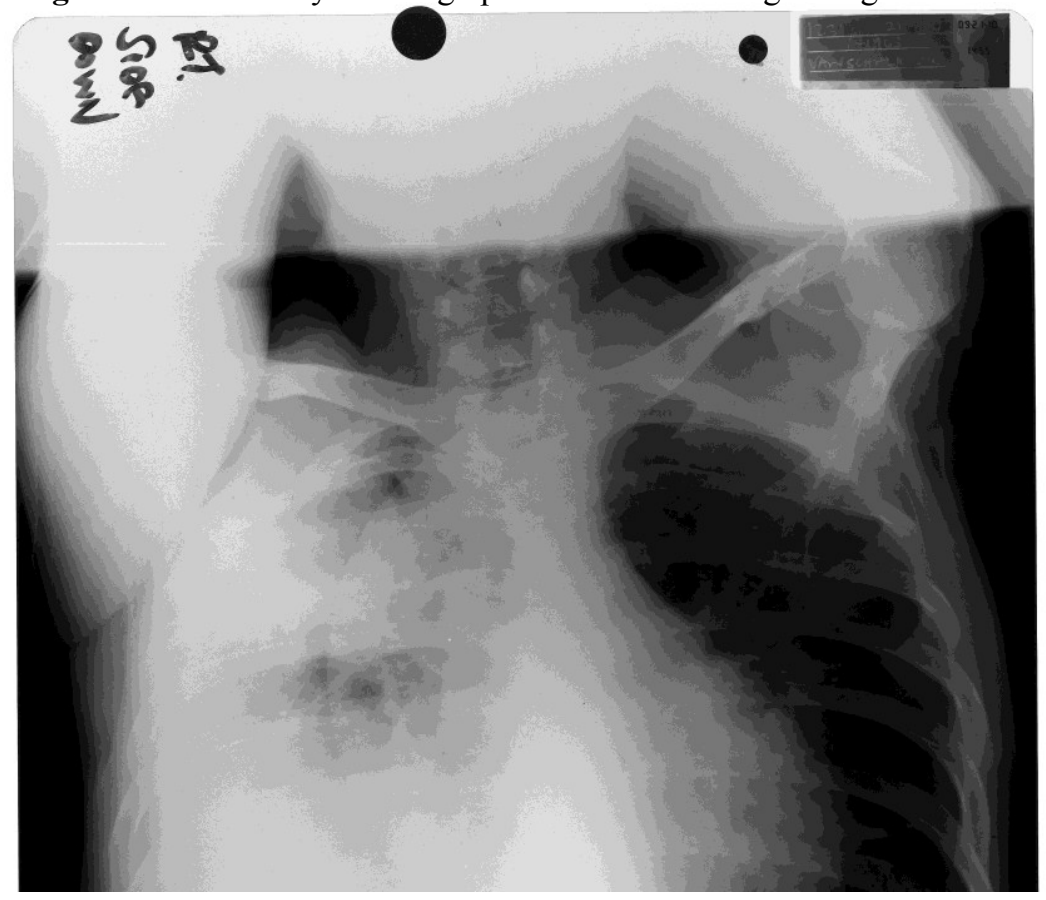

organism is an aerobic Gram-negative bacillus which, unlike Bordetella pertussis and Bordetella parapertussis, grows readily on media used for routine microbiological isolation. It is motile and positive for oxidase, catalase and urease. In the evaluation of the colorimetric Vitek II card for identification of Gram-negative rods, Zbinden et al. [10] concluded that any species other than Achromobacter xylosoxidans, Acinetobacter species, Burkholderia cepacia group, Pseudomonas aeruginosa and Stenotrophomonas maltophilia should undergo 16SrRNA gene sequence analysis if accurate identification is required in spite of accurate identification on the colorimetric Vitek II card. Our organism was initially identified using the Vitek II colorimetric card, which showed excellent identification ( $99 \%$ confidence). This identification was later confirmed by $16 \mathrm{SrRNA}$ gene sequence analysis [11].

The standard method for susceptibility testing has not been established and discrepancies between in vitro susceptibility and response to therapy has been previously described [4]. A study done by Woolfrey and Moody [1] comparing the standard broth microdilution method with Vitek antimicrobial susceptibility testing showed discrepancies between these two methods on many antibiotics (ampicillin, carbenicillin, ticarcillin, cephalosporins, and trimethoprim-sulfamethoxazole). Ciprofloxacin, however, showed full susceptibility with both methods. It is therefore recommended that Vitek susceptibility testing be confirmed by another method. The appropriate therapy for $B$. bronchiseptica infection has not been ascertained, but the organism is usually susceptible to aminoglycosides, antipseudomonal penicillins, broad spectrum cephalosporins, chloramphenicol and tetracyclines [1]. Our patient was allergic to penicillin and was therefore treated with ciprofloxacin. The cause of death was not clear and a postmortem was not performed, but it could have been either related to the infection or to the underlying poor general condition of the patient.

This case illustrates the potential virulence of $B$. bronchiseptica in susceptible patients and to our knowledge; it is the first case of primary peritonitis due to this organism.

\section{Acknowledgments}

We thank the Department of Medicine and the staff at the Microbiology laboratory, Addington Hospital, Durban. We also thank Dr Tumelo Mabesa, Department of Radiology, Addington Hospital and Dr Victoria Howell, Department of Microbiology, Inkosi Albert Luthuli Central Hospital. 


\section{References}

1. Woolfrey BF, and Moody JA (1991) Human infection associated with Bordetella bronchiseptica. Clin Microbiol Rev 4: 243-255.

2. National Center for Biotechnology Informationhttp://blast.ncbi.nlm.nih.gov/Blast.cgi. Accessed 11 July 2011.

3. Dworkin MS, Sullivan PS, Buskin SE, Harrington RD, Olliffe J, MacArthur RD, Lopez CE (1999) Bordetella bronchiseptica infection in human immunodeficiency virusinfected patients. Clin Infect Dis 28: 10951099

4. Won K, Ha G, Tak W,Kim JJ, Kang H (2009) Relapsing peritonitis caused by Bordetella bronchiseptica in continuous ambulatory peritoneal dialysis patient: a case report. J Korean Med Sci. 24: S215-218.

5. Ghosh HK and Tranter J (1979) Bordetella bronchicanis (bronchiseptica) infection in man: review and a case report. J Clin Pathol 32: 546-548.

6. Conn HO and Fessel JM (1971) Spontaneous bacterial peritonitis in cirrhosis: variation on a theme. Medicine (Baltimore) 70: 161-197.

7. Lahnborg G, Friman L, Berghem L (1981) Reticuloendothelial function in patients with alcoholic liver disease. Scand J Gastroenterol 16: 481-489.

8. Byrd LH, Anama L, Gutkin M, Chmel H (1981) Bordetella bronchiseptica peritonitis associated with continuous ambulatory peritoneal dialysis. J Clin Microbiol 14: 232233.

9. Hadley K, Torres MA, Moran J, Schiller B (2009) Bordetella bronchiseptica Peritonitis-beware of the dog. Perit Dial Int 29: 670-678.

10. Zbinden A, Böttger EC, Bosshard PP, and Zbinden R (2007) Evaluation of the Colorimetric VITEK II Card for identification of Gram-negative nonfermentative rods: comparison to $16 \mathrm{~S}$ rRNA gene sequencing. J Clin Microbiol 45: 2270-2273.

11. Clarridge JE, III (2004) Impact of 16S rRNA gene sequence analysis for identification of bacteria on clinical microbiology and infectious diseases. Clin Microbiol Rev 17: $840-862$

\section{Corresponding author}

Dlamini Nomonde Ritta

PO Box 1316

Wandsbeck 3629

Durban, South Africa

Telephone: 0824154300; Fax: 0866513617

Email: Nomonde.dlamini@nhls.ac.za

Conflict of interests: No conflict of interests is declared. 